\title{
OPORTUNIDADES Y DESAFÍOS DE LA INVESTIGACIÓN EN TURISMO EN PATAGONÍA
}

\author{
OPPORTUNITIES AND CHALLENGES ABOUT TOURISM RESEARCH IN PATAGONIA
}

\author{
Pablo R. Szmulewicz ${ }^{1} \&$ Katerina M. Veloso ${ }^{2}$
}

\begin{abstract}
The tourism sector in Chile has had a considerable increase over the last 10 years and a significant change in the visitors' interests focus. On the one hand, the arrival of international visitors increased from $1,700,000$ to over $3,000,000$ in 2011. On the other hand, tourists show a growing motivation for different kind of special interest tourism, mainly in the development of activities in rural and natural areas. In fact, nowadays, more than half of international tourists that visit our country are motivated by performing activities related to nature and outdoor sports, which explains the fact that over the last years visits to wildlife protected areas in the country have increased considerably.

The enactment of new public policies and the approval of the Tourism Law indicate the social and economic salience that the tourism sector is achieving. These policies have encouraged innovation and research development, by increasing the funds that drive new businesses, new products, the development of tourist destinations and the cientific knowledge generation about the sector.

However, despite the rapid growth the tourism industry has had in Chile, there are still challenges to be fulfilled, mainly refering to the generation of tourism scientific knowledge and its difussion and transfer to potential applicants. Between various universities and $\mathrm{R} \& \mathrm{D}$ centres that provide tourism programs in Chile, just a few of them do tourism research and an important part is produced by the National Tourism Agency and Universities and research centers without tourism training programs.

In the southern zone of Chile this problem is particularly stronger, because even though this region has the highest potential for tourism development, it does not have any tourism training and research programs.

The national priority assigned to the Special Interest Tourism, in which the Magallanes' Region presents extraordinary comparative advantages, offers to the University of Magallanes the opportunity of building a Centre for Research, Development and Innovation in Tourism in the Patagonia. This Centre would aim to generate interdisciplinary and applied research that would contribute to consolidate the Patagonia as priority international destination.
\end{abstract}

Key word: tourism research, special interest tourism, Patagonian, applied research.

\footnotetext{
${ }^{1}$ Instituto de Turismo, Universidad Austral de Chile, casilla 567 Valdivia, Chile. Profesor Visitante, Universidad de Magallanes. pablo.szmulewicz@gmail.com.

${ }^{2}$ Instituto de Turismo de la Universidad Austral de Chile. Casilla 567, Valdivia, Chile. katerinaveloso@gmail.com
} 


\section{RESUMEN}

El sector Turismo en Chile ha tenido un considerable aumento durante los últimos 10 años y un cambio sustancial en el foco de los intereses de los visitantes. La llegada de visitantes internacionales aumentó de 1.700.000 a más de 3.000.000 en el año 2011. Por otra parte, se aprecia una creciente motivación de los turistas por las distintas modalidades del turismo de intereses especiales, principalmente por el desarrollo de actividades en áreas rurales y naturales. De hecho, actualmente, más de la mitad de los turistas de larga distancia que visitan nuestro país lo hacen motivados por la realización de actividades relacionadas con la naturaleza y deportes al aire libre, lo que explica que durante los últimos años las visitas a áreas silvestres protegidas del país han aumentado considerablemente.

La generación de nuevas políticas públicas para el sector y la promulgación de la Ley de Turismo, son hechos que indican también la relevancia social y económica que el sector está alcanzando. Estas políticas han favorecido el desarrollo de la innovación y la investigación, con el aumento de fondos que impulsan nuevos emprendimientos, nuevos productos, el desarrollo de destinos turísticos así como la generación de información científica del sector.

Sin embargo, a pesar de este rápido crecimiento que ha logrado el sector turístico en Chile aún existen desafíos que cumplir principalmente en el ámbito de la generación de conocimiento científico sobre el sector y la difusión y transferencia de las investigaciones a los potenciales interesados. De las diversas universidades y centros de I+D que tienen programas de turismo en Chile, sólo algunos realizan actividades de investigación en turismo y una parte importante de la investigación es generada por el Servicio Nacional de Turismo y por universidades y grupos de investigación que no cuentan con programas de formación en turismo.

Esta carencia es especialmente grave en la zona austral ya que siendo una región con el más alto potencial de desarrollo turístico no cuenta con programas de formación e investigación en turismo. La prioridad nacional al desarrollo del Turismo de Intereses Especiales, en que la Región de Magallanes cuenta con extraordinarias ventajas comparativas genera una oportunidad para que la Universidad de Magallanes se encamine a la creación de un Centro de Investigación, Desarrollo e Innovación en Turismo de la Patagonia, orientado a generar investigación aplicada e interdisciplinaria que contribuya a consolidar Patagonia como destino internacional prioritario.

Palabras clave: investigación turística, turismo de intereses especiales, Patagonia, investigación aplicada.

\section{SITUACIÓN ACTUAL DEL SECTOR TURISMO EN CHILE.}

Importancia económica del sector Turismo en Chile

Durante los últimos 10 años la llegada de turistas extranjeros a Chile ha aumentado considerablemente, logrando el país constituirse en el 3er destino de Sudamérica. En el año 2001 arribaron poco más de 1.700.000 turistas y actualmente se superan los 2.500.000. La demanda de turismo en Chile, en 2008, tuvo el mayor crecimiento en América Latina con 8,5\% y se espera un crecimiento del $6,4 \%$ promedio anual en la década estimándose que para el año 2014 llegarán más de 3 millones de visitantes.

Durante el año 2009 el país tuvo un ingreso de US\$ $1.977,8$ millones generado por el gasto que realizaron los visitantes extranjeros, cifra que incluye el desembolso hecho tanto por turistas como por excursionistas, y lo generado por transporte internacional de aquellos visitantes que utilizaron medios nacionales (INE - SERNATUR, 2010).

El sector aporta aproximadamente el $3,07 \%$ del PIB y genera alrededor del $2 \%$ de la fuerza de trabajo. Al comparar los rubros de exportación más relevantes del país es posible señalar que los ingresos de divisas por turismo receptivo representan el $7,2 \%$ de los ingresos por exportaciones de cobre; el $65,7 \%$ de los provenientes del sector frutícola; el $97,5 \%$ del salmón y la trucha; el $75,6 \%$ de las exportaciones de celulosa, papel y otras; y superan a los productos forestales, muebles y madera en un $28,6 \%$ y al vino en un 43,2\% (CORFO, 2008) (ver Fig. 1). 


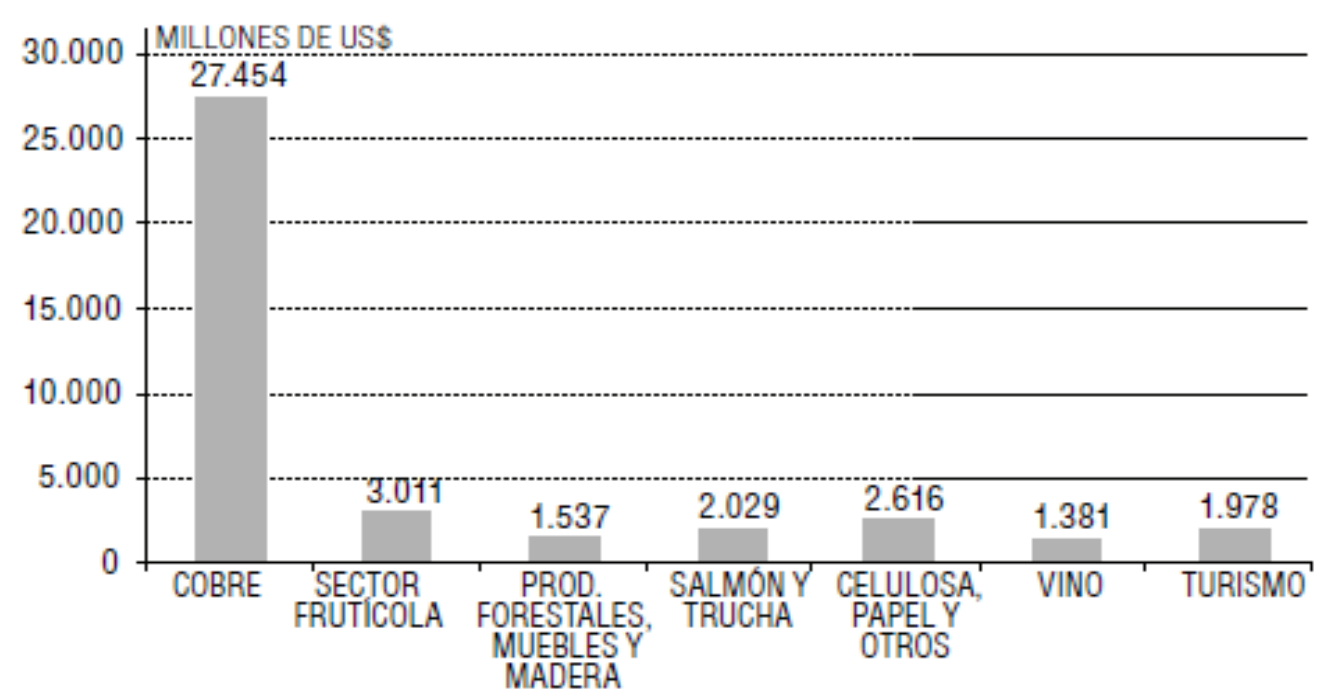

Fig. 1. Exportación de Bienes y Turismo en Chile, 2009. Fuente: Banco Central de Chile, 2009 (en INE-Sernatur, 2010).

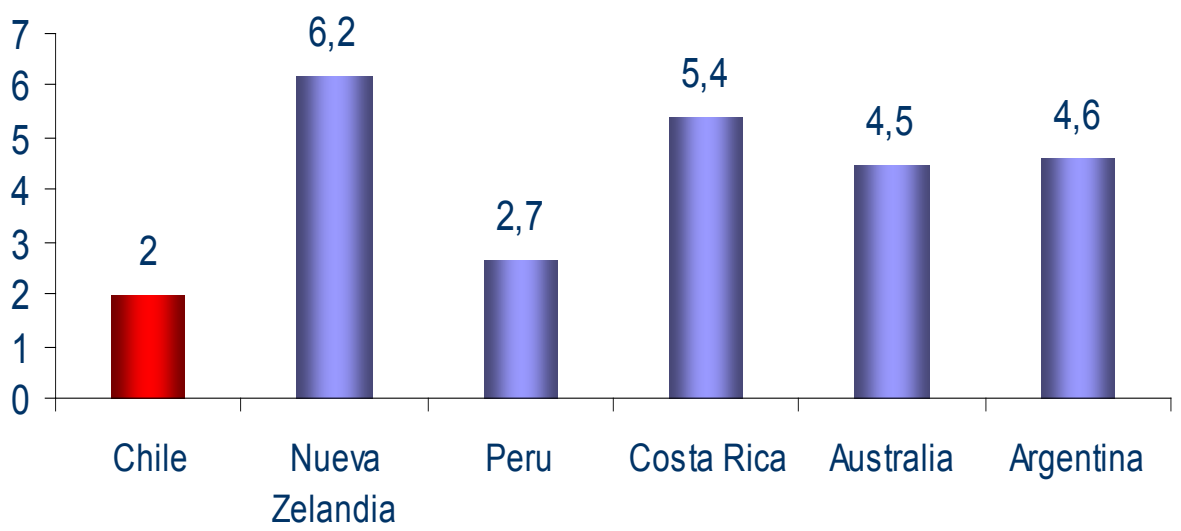

Fig. 2. Empleo Turístico como porcentaje del total del empleo. Fuente: WEF, 2009.

El aporte del turismo receptivo equivale al $3,7 \%$ del total de las exportaciones de bienes y al $23,3 \%$ del total de las exportaciones de servicios. Sin embargo, el sector es todavía una actividad emergente, que no responde a su importancia en términos de potencialidad lo que queda de manifiesto al observar que su contribución a la economía es menor que la de países referentes (ver Fig. 2).
Turismo en Áreas Rurales y Naturales en Chile

La creciente importancia del Turismo en Áreas Rurales y Naturales en el mundo, en particular del Turismo de Intereses Especiales (en adelante TIE), impulsado por el aumento de la demanda de actividades de recreación al aire libre, ha generado el desarrollo de nuevos tipos de turismo, basados en 
el aprovechamiento del medio natural y, también, en estrecho vínculo con el patrimonio cultural. La motivación por los atractivos naturales ha incrementado significativamente la cantidad de visitantes y elevado las tasas anuales de crecimiento turístico. Este aumento, aunque positivo desde la perspectiva económica, plantea al turismo un desafío de magnitud para asegurar su sustentabilidad ambiental, cultural y socio económica, en el mediano y largo plazo.

En Chile durante la última década, se ha observado una demanda creciente por el desarrollo del turismo en áreas rurales y naturales. El país cuenta con atractivos y destinos turísticos singulares que le otorgan ventajas competitivas en el mercado del turismo de intereses especiales (agroturismo, turismo de naturaleza, turismo científico, turismo del vino, turismo arqueológico, astronómico, entre otros) permitiendo su posicionamiento turístico internacional en torno a los conceptos de naturaleza, cultura y actividades outdoors (CORFO, 2008). Más de la mitad de quienes visitan Chile viajan influidos por los paisajes naturales: Selva Valdiviana, Desierto de Atacama, Patagonia, Torres del Paine, Tierra del Fuego, Isla de Pascua, Andes, entre otras, son marcas e iconos que guardan un enorme potencial turístico. De hecho, el 56\% de los extranjeros que visitan nuestro país realizan actividades relacionadas con la naturaleza y el deporte, como trekking, andinismo, deportes náuticos, entre otros. Otra expresión de este significativo desarrollo del TIE, se aprecia en las visitas a Parques Nacionales y otras categorías de áreas de conservación. En el período 2000-2010, las áreas protegidas públicas de Chile registraron un incremento del $56 \%$ en el ingreso de visitantes, alcanzando un total de 1.816 .916 personas el año 2010, a diferencia del año 2000 que alcanzó 1.022 .002 de visitas.

\section{TURISMO Y POLÍTICAS PÚBLICAS DE DESARROLLO ECONÓMICO EN CHILE}

Turismo en la Política Nacional de Innovación y Clusters

Desde el Ministerio de Economía de Chile se ha impulsado una política de desarrollo económico con eje en Clusters productivos prioritarios, para, en el marco de las políticas de innovación, consolidar un conjunto de sectores de alto potencial económico y centrar los esfuerzos de innovación productiva en esos clusters. Fundamentado en un estudio internacional contratado al Boston Consulting Group, el Gobierno de Chile definió los clusters (grupos o subsectores económicos) que pueden tener mayor potencial de crecimiento y cuyo esfuerzo para lograr la competitividad nacional e internacional es menor. El sector turismo tradicional ha quedado ubicado en una posición relativamente baja en esta suerte de ranking. Sin embargo, el denominado "turismo de intereses especiales": turismo de nichos, asociado fundamentalmente a naturaleza, cultura, actividades, deportes, desarrollo personal, negocios, etc. ha sido considerado entre los clusters prioritarios y está ocupando una posición protagónica en el conjunto de los sectores que el país va a consolidar (CORFO, 2008).

Turismo en la Política de Desarrollo Económico Territorial

En Chile se ha impulsado una política de desarrollo económico-territorial a través de un programa, desde el nivel nacional, que abarca todo el conjunto de los municipios y de las regiones, denominado programa Chile Emprende. Este programa de desarrollo económico en territorios supramunicipales, ha tenido como objetivo potenciar el fortalecimiento de las micro y pequeñas empresas, estableciendo sinergias y esfuerzos conjuntos público-privados en territorios con vocaciones productivas similares. El programa operó entre los años 2001 y 2010 y contó con el apoyo de un conjunto de organismos públicos de diferentes ámbitos, que formaron el entramado de desarrollo territorial. Los gobiernos locales tienen un rol significativo, aunque bastante secundario, al formar parte de una estructura de dirección compuesta por servicios regionales y centralizados junto a las organizaciones representativas del sector privado. Por lo tanto, hay una política de gobierno que impulsa a los municipios a trabajar en el desarrollo económico territorial en coordinación con un conjunto de otros organismos públicos y también en conjunto con otros municipios. Este programa constituye un avance hacia una configuración supramunicipal. El sector turístico tuvo un papel destacado como uno de los sectores ejes en casi todos los territorios de aplicación del programa. 
Políticas Públicas del sector Turismo

Chile, ha planteado una Política Nacional de Turismo, que se basa fundamentalmente en dos elementos: la promulgación de la Ley Marco de Turismo y la estructuración de un Programa de Fomento. El marco legal lo constituye la Ley de Turismo promulgada el 4 de febrero del año 2010, que sienta las bases para la regulación del sector, poniendo énfasis en el establecimiento de Zonas de Interés Turístico (ZOIT), promoviendo el desarrollo turístico en las Áreas Silvestres Protegidas, el mejoramiento de la Promoción Internacional de Chile, la mayor protección de los Derechos de los Turistas, además de certificar la calidad de los servicios que entrega el país.

Ley de Turismo de Chile

Los Principios orientadores de la Ley de Turismo de Chile son según Szmulewicz et al. 2008:

a) Coordinación e integración normativa y ejecutiva de los distintos organismos públicos y privados relacionados directa o indirectamente con la actividad turística.

b) Contribuir al aprovechamiento del tiempo libre y la revalorización de la identidad cultural de las comunidades.

c) Desarrollo del turismo en armonía con los recursos naturales y culturales.

d) Creación de productos y servicios competitivos, optimización de la organización y gestión de las empresas, homologación de estándares de calidad y transparencia del mercado.

e) Asegurar una adecuada oferta de servicios y lograr rentabilidad empresarial para la sostenibilidad.

f) Materializar sus potenciales efectos en la descentralización que posee el desarrollo turístico.

En relación con la institucionalidad pública, se crea una instancia superior de coordinación en materia de turismo: el Consejo de Ministros del Turismo. En materia de gestión de territorios con aptitud turística, la ley establece principalmente cuatro innovaciones. La primera, es elevar la declaración de Zonas de Interés Turístico a un Acuerdo del Consejo de Ministros, estableciendo que las ZOIT tendrán prioridad para la ejecución de programas y proyectos de fomento del desarrollo turístico, así como en la asignación de recursos para la imple- mentación de infraestructura y equipamiento para el turismo. La segunda es la posibilidad de utilizar Áreas Silvestres Protegidas del Estado para desarrollar el turismo con la autorización del Consejo de Ministros del Turismo, del Ministro que administra estas áreas y del Ministro de Bienes Nacionales. Éste último, además, otorgará las concesiones para usos e infraestructura turística, por medio del Sistema de Concesiones Fiscales establecido en los artículos 57 a 63 del Decreto Ley 1.939 de 1977. Un tercer cambio es crear el Comité Nacional de Promoción Turística, que será responsable de contratar planes de marketing, realizar estudios de mercado, proponer la política y los programas de promoción turística y velar por su cumplimiento. El Servicio Nacional de Turismo (SERNATUR) será responsable de la ejecución de las actividades de promoción. El cuarto cambio sustancial es establecer un Sistema de Clasificación, Calidad y Seguridad de los Prestadores de Servicios Turísticos, que comprende un registro de los servicios turísticos y la certificación, de carácter voluntario, con un Sello de Calidad y/o Seguridad Turística otorgado por SERNATUR (Szmulewicz et al. 2008).

\section{Programas Públicos de Fomento al Turismo}

Como parte fundamental de las políticas de fomento del turismo se crea el Programa Innova Chile al alero de Corfo. Este programa, generó un amplio conjunto de proyectos de apoyo al sector y dio un salto cuantitativo trascendente al materializar 100 proyectos vinculados al cluster TIE sólo en el año 2008 por un monto de $\$ 7.902$ millones. Se aprueban además otras líneas como las Agendas de Innovación, los programas de Transferencia y Difusión tecnológica y Nodos Tecnológicos. Otras líneas donde el sector turismo encuentra importantes recursos son las de Formación de Capital Humano Avanzado, Capital semilla y Prospección de mercados. La curva de crecimiento de los fondos de Corfo destinados a la Investigación e Innovación es exponencial (CORFO, 2008) (ver Fig. 3).

Tal como aparece en la Fig. 4, en cuanto a la distribución territorial de estos recursos las regiones de Valparaíso, con más de 1.000 millones de recursos adjudicados, la Araucanía, Magallanes, Aysén y Antofagasta son las que han recibido mayor cantidad de recursos de Corfo para el desarrollo de 


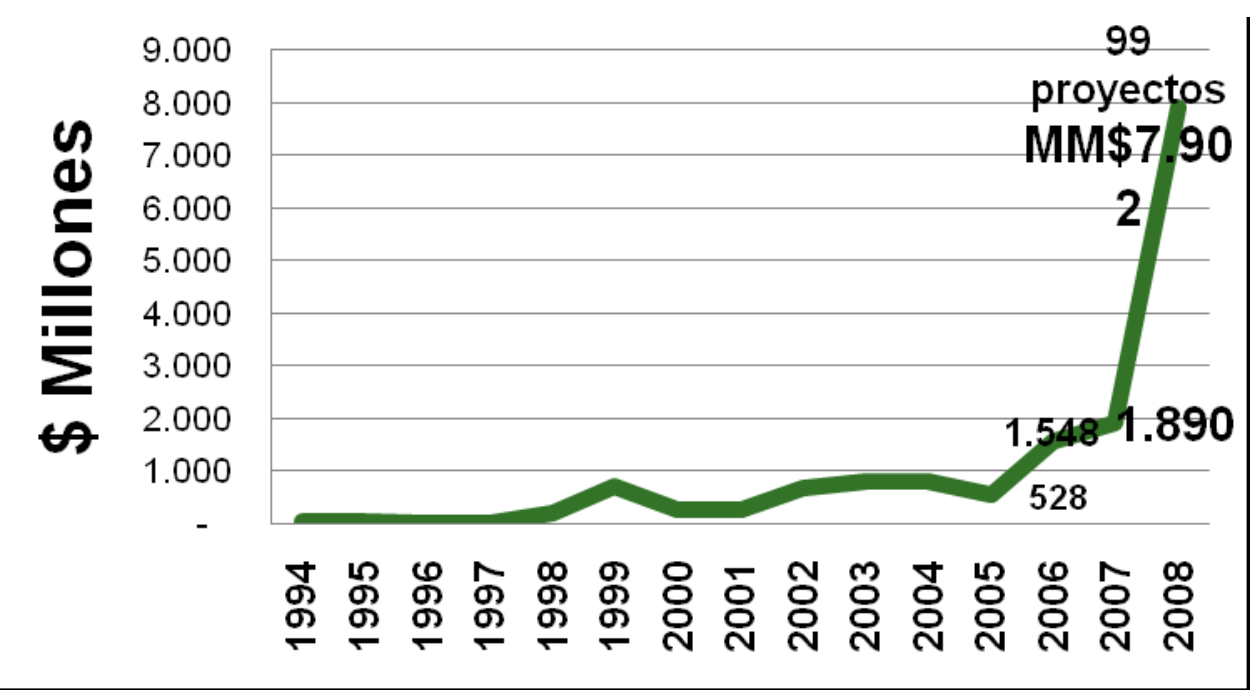

Fig. 3. Fondos del Programa de Innovación en Turismo de Corfo. Fuente: Corfo, 2008.

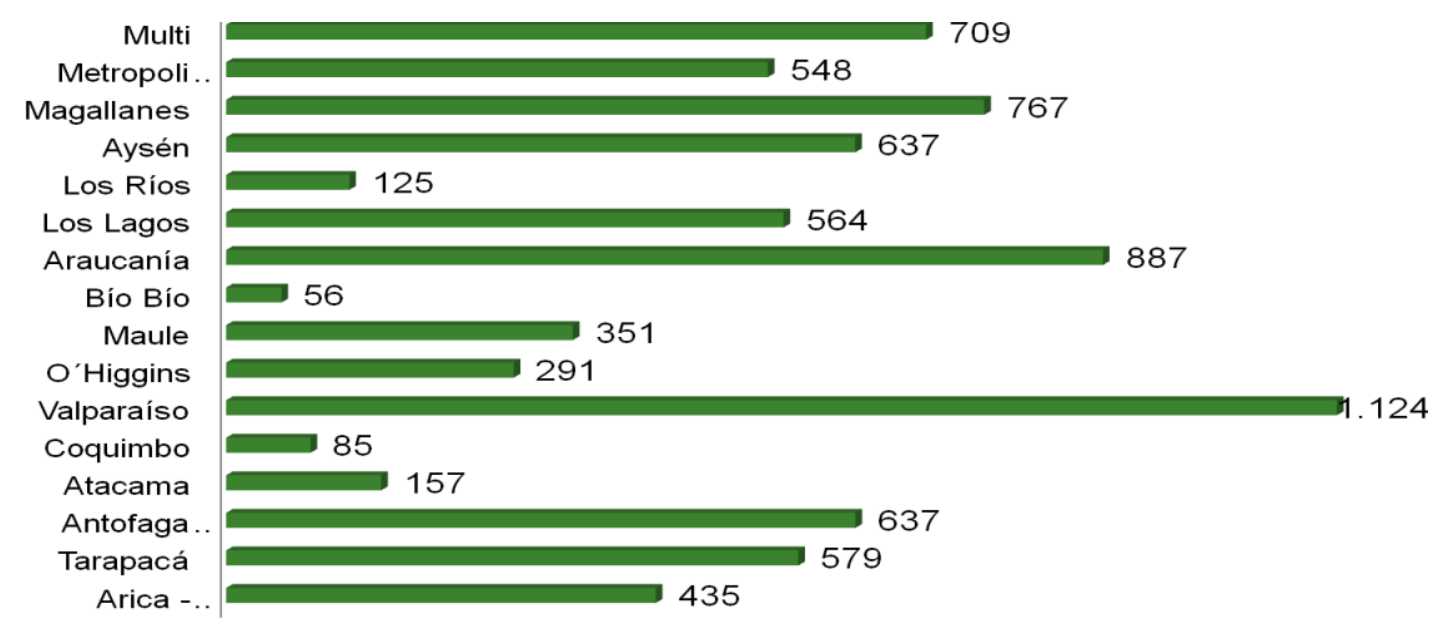

Fig. 4. Distribución territorial de los fondos para turismo de Corfo, 2008 . Fuente: Corfo, 2008.

proyectos turísticos. Sin duda que, en comparación con su población, la región de Magallanes es la que se presenta como zona prioritaria para la colocación de fondos nacionales de fomento del turismo, aún sin considerar los aportes de los fondos regionales que también son altamente significativos en su empuje al sector (CORFO, 2008).

\section{DESAFÍOS DE LA INVESTIGACIÓN Y LA INNOVACIÓN EN TURISMO EN CHILE}

Estado de la Investigación Turística en Chile
En términos generales, el desarrollo de la investigación turística en el país es todavía muy escaso. No obstante, los recursos para la investigación turística han aumentado con el paso del tiempo, a la par con el interés creciente de las autoridades por el tema, tal como se ha señalado en el apartado anterior.

En Chile existen actualmente 13 universidades que cuentan con Programas de formación en Turismo y otros tantos Institutos Profesionales que imparten carreras profesionales o técnicas de Turismo. Sin embargo, de todas estas instituciones sólo algunas: Universidad Austral de Chile, Universidad de Ma- 
gallanes, Universidad de la Serena, Universidad de Valparaíso, Universidad Tecnológica Metropolitana, Universidad Arturo Prat, Universidad Andrés Bello, han desarrollado actividades de investigación.

Gran parte de la investigación en Turismo en Chile ha sido realizada por el mismo Organismo Oficial de Turismo de Chile (SERNATUR). Otra parte significativa de la investigación turística ha sido desarrollada por universidades que no poseen programas de formación en Turismo, sino equipos investigadores que trabajan en diversas áreas de la realidad regional y que cubren estudios del sector Turismo. Tal es el caso de la Universidad Católica de Valparaíso y su grupo de Geografía, la Universidad de la Frontera y su Laboratorio de Ordenamiento Territorial en Temuco, la Universidad de Concepción y su centro EULA, la Universidad de Talca con su Observatorio Turístico y últimamente el Centro de Investigación en Ecosistemas de la Patagonia (CIEP), el Centro de Estudios del Cuaternario (CEQUA), la Fundación OMORA, entre otras. Estos grupos de investigadores, dado su carácter de multipropósito, no realizan esfuerzos sistemáticos y permanentes en el campo del turismo y tampoco tienen la necesaria vinculación con el resto de los actores del turismo nacional. La investigación turística, en estos casos, no responde aun a un programa orgánico de las universidades o los centros de investigación. Por lo anterior, se puede deducir que la participación de la Universidad en la investigación turística en Chile es todavía muy baja.

El financiamiento de las labor de I+D en turismo se concentra en los aportes públicos, siendo el aporte privado muy escaso y el universitario bastante bajo. Todos los recursos provienen de fondos concursables nacionales o sectoriales, fondos especiales de apoyo a empresas, etc.

Hasta ahora, se puede decir que la mayoría de los estudios turísticos efectuados en Chile tienen un carácter exploratorio y descriptivo. Se ha logrado un cierto rigor metodológico, pero reducido a un escaso número de investigadores en el área. Los estudios realizados se pueden agrupar en: investigaciones sobre los recursos turísticos; investigaciones sobre el comportamiento de la demanda y las características de la comercialización; estudios sobre los movimientos turísticos y análisis de la oferta de servicios turísticos. No se puede dejar de señalar la gran importancia de la información estadística en turismo, ya que buena parte de los datos de fuentes primarias deben estar en estas bases. Las deficiencias de la información estadística son muy grandes y notorias. Recién en los últimos años se están materializando iniciativas para mejorar la información estadística; pero la carencia de técnicos y de personal para aplicarse al sector turismo debe todavía ser superada (Szmulewicz, 1998).

Generalmente las investigaciones se realizan en equipos multidisciplinarios que, dependiendo del tema, están integrados por Estadísticos, expertos en Computación, Geógrafos, Antropólogos, Economistas y Ecólogos entre otros. Demás está decir que la interdisciplinariedad es, además, una exigencia propia del fenómeno turístico, social e integral por naturaleza y es también una vía para aprovechar racionalmente los escasos recursos disponibles.

Pendiente se encuentra la tarea de recuperar la "memoria colectiva" de la investigación turística con el objeto de establecer los hitos existentes, como asimismo, difundir estudios que, a pesar de no poseer actualidad en los datos, pueden servir de antecedentes para la futura marcha de la investigación.

La conversión de los estudios en publicaciones científicas es otra dificultad encontrada. El espíritu de divulgación no siempre va de la mano con el del investigador, por lo que el volumen de publicaciones turísticas en Chile es aún escaso. Sólo se dispone de una revista especializada: Gestión Turística, editada por el Instituto de Turismo de la Universidad Austral de Chile. Cabe mencionar acá que un buen número de artículos sobre turismo se publican en revistas de otras disciplinas como Geografía, Economía, Administración, Arquitectura, etc.

En general la investigación turística en las universidades chilenas tiene una estrecha relación con el medio productivo. Esto no puede ser de otra forma, ya que gran parte del financiamiento para la investigación proviene de organismos públicos que inducen a tratar temáticas atingentes a los problemas del sector.

Fortalecimiento de la Investigación Turística en Chile

El año 2006 marca un hito en el desarrollo de la ciencia sobre el turismo en Chile, con el nacimiento de la Sociedad de Investigadores en Turismo de Chile (en adelante SOCIETUR). Esta 
organización es una Corporación de Derecho Privado, sin fines de lucro, de carácter estrictamente académico y científico que tiene como su área de interés el fenómeno del turismo y todo cuanto diga relación con dicha actividad, tanto desde la perspectiva social, cultural y económica, como desde las ciencias ambientales, la historia, el derecho y todas aquellas disciplinas que abordan temáticas relacionadas con los viajes, el desplazamiento de turistas y las actividades destinadas a brindar servicios para su adecuado desarrollo, según rezan sus propios estatutos. Su objetivo principal es elevar la calidad de la investigación científica en este campo, lograr la articulación público - privada para el conocimiento $y$ constituirse en un referente científico consultivo para el desarrollo del turismo en Chile.

SOCIETUR integra, al año 2011, más de 50 destacados investigadores de universidades con programas de Turismo, centros de investigación y de algunas instituciones públicas. Dentro de las actividades más destacadas que ha desarrollo SOCIETUR se encuentra la realización de 5 congresos anuales de investigación y 4 foros nacionales de turismo, la publicación de 3 libros con los Anales de sus congresos y con el apoyo del SERNATUR se llevó a cabo, en 2 versiones, el Concurso de Tesis de Pre y Postgrado en Turismo, que ha permitido estimular la investigación de los jóvenes en el sector turístico. Cuenta también con convenios de colaboración con otras Asociaciones Científicas de Turismo internacionales, como la de Brasil, y con otras agrupaciones del sector a nivel nacional. SOCIETUR además ha entregado apoyo en la formación de la Asociación de Profesionales en Turismo de Chile (APROTUR).

Lecciones de la crisis económica y el desastre natural en Chile para la investigación turística

El año 2010 la crisis económica mundial golpeó al sector. El turismo mundial disminuyó un $4 \%$ y en las Américas la disminución alcanzo el 5\% (World Economic Forum, 2009). A nivel nacional para el mismo año se estimó en cerca de un $11 \%$ la disminución de la llegada de turistas internacionales. Esta disminución se debió a diversos factores externos como la gripe A (H1N1), la crisis económica que afecta a países emisores de turismo como España, Italia, Francia, Alemania, entre otros, y el terremoto del 27 de febrero que afectó una extensa zona del centro de Chile, principalmente por la destrucción de zonas de recreación y turismo y los deterioros ocasionados en el único aeropuerto internacional del país.

Como bien señaló el Presidente de las Sociedades Científicas de Chile, el sismo del 27 de febrero había sido pronosticado y los estudios al respecto se habían publicado en revistas científicas internacionales. Con esto ha quedado de manifiesto que una de las principales fallas de la investigación científica en Chile, es la escasa transferencia del conocimiento generado a la sociedad, a las autoridades y actores relevantes, y a todos quienes pueden hacer uso de la información para una mejor toma de decisiones, asegurando la calidad de las políticas y los programas públicos y con ello disminuir el riesgo de las inversiones públicas y privadas. Otras falencias que fueron evidentes en esta catástrofe natural, fueron la falta de un adecuado sistema de prevención; la ausencia de planes de contingencia que indicarán que hacer en caso de sismos; la inexistencia de una red de monitoreo que permitiera conocer la magnitud del movimiento y la carencia de sistemas de comunicaciones eficientes con la potencia necesaria para operar en caso de emergencias. En parte, estas debilidades de la investigación científica y sobre todo de su vinculación con los actores públicos, fue responsable de las dramáticas consecuencias para las vidas humanas y los bienes materiales del terremoto del $27 \mathrm{~F}$.

\section{Orientaciones de la Investigación Turística en Chile}

Algunas de las orientaciones que, a nuestro juicio, deben guiar el desarrollo de la investigación turística en Chile son:

1. Respeto por la identidad y la cultura local: este principio se traduce en la máxima consideración, de parte de los investigadores, a las normas nacionales y locales y aceptación de las autoridades regionales propias de cada comunidad con la que se colabora. El Código del Turismo señala que los agentes del desarrollo turístico prestarán atención a las tradiciones y prácticas sociales y culturales de todos los pueblos, incluso a las de las minorías nacionales y de las poblaciones autóctonas, y reconocerán su riqueza (OMT, 2001:8).

2. Búsqueda de la verdad y el mayor rigor 
científico posible: se busca la verdad a través del método científico, de la vinculación efectiva con la realidad territorial y empresarial del sector, comunicando las características y potencialidades efectivas que tienen los destinos y las comunas así como potenciar el desarrollo turístico en zonas con verdadera vocación y presentar con claridad y responsabilidad los mensajes que los investigadores transmiten.

3. Pertinencia: valor que debe ser entendido como reconocimiento al esfuerzo y la dedicación especializada de los distintos actores sociales. A pesar de que todas las opiniones sean tomadas en cuenta, sin duda hay personas que conocen mejor el territorio que otras, y hay actores que conocen más un ámbito que otro. Hay que tener una consideración especial por la idoneidad de cada actor en su respectiva temática.

4. Sentido Formativo: contribuyendo a formar capacidades propias, entregar metodologías y convertir el proceso de diagnóstico, planificación y gestión turística en una experiencia educativa.

5. Descentralización: un valor relevante de las actividades de I+D es fomentar la descentralización y la delegación del poder. En todas las regiones, existe un marcado centralismo, que queda reflejado en que la gran mayoría de las decisiones estratégicas son tomadas a nivel cupular y muchas veces sin considerar las opiniones y visiones locales. En este aspecto, como una forma de descentralización y desconcentración el objetivo es que, se distribuyan las acciones y las decisiones con la mayor amplitud territorial, priorizando también proyectos que están en áreas más alejadas pero con un alto potencial que crecimiento (Szmulewicz, 2011).

6. Búsqueda de la Excelencia: que significa acuerdos de colaboración no sólo para la fase de diagnóstico, sino para el tratamiento que implica un alto compromiso con el logro de los objetivos y el cumplimiento de las metas en cada proyecto que se asume. Los Centros de Investigación en Turismo se deben comprometer en un programa de desarrollo en una comunidad, a mediano y largo plazo. No sólo se orientan a realizar proyectos puntuales $y$ al concluir el plazo de su desarrollo se retiran, sino que se comprometen a seguir una línea de trabajo durante el tiempo suficiente para materializarlos proyectos (Szmulewicz y Veloso, 2012).

7. Construir sobre lo construido: se valora generar conocimiento sobre lo que ya se ha cons- truido. Esto obliga a recoger ampliamente lo que ya se ha estructurado, escrito, publicado y pensado en un campo de investigación y la zona en estudio. Esta posición exige ser coherentes con las estrategias regionales de desarrollo y con las políticas nacionales, regionales y locales. Implica iniciar toda investigación con el "Estado del arte", sistematizando el conocimiento sobre el área y sobre el proyecto y convocar a actores experimentados en el tema y en el territorio.

8. Colaboración internacional y Prioridad a los recursos endógenos: los convenios entre universidades y las relaciones personales entre investigadores, la participación en redes a través de Internet, es un desafío que no es posible seguir postergando. La cooperación internacional en el campo de la investigación, tanto entre países con diversos grados de avance, como entre los mismos países latinoamericanos, surge como una necesidad urgente del desarrollo del Turismo.

Sin duda que es importante recoger las experiencias internacionales, pero también hay que adaptarlas a la realidad nacional y regional, considerando las características propias de los destinos a desarrollar. Es fundamental tener la convicción de que es posible encontrar recursos en el territorio, otorgarle prioridad a aprovechar tanto su capital social, como especialmente su capital humano.

9. Transparencia y Honestidad: es importante la existencia de reglas claras, el conocimiento de los procesos y procedimientos, el predominio del sistema de concursos y el conocimiento público de todas las oportunidades que existen. Hay que seguir trabajando en disminuir al máximo la corrupción, no solo en las decisiones políticas, sino que incluso en las decisiones científicas, las educativas y las de desarrollo económico muy especialmente.

10. Evaluación: es indispensable estar sometidos, no sólo a una autoevaluación, si no que valorar el proceso objetivo de recoger opiniones sobre el trabajo del investigador y recoger también la valoración que hacen los agentes públicos y privados sobre los esfuerzos que se realizan. Este principio obliga a tener una mirada crítica sobre los procesos y las acciones del investigador.

11. Visión de Futuro: una componente significativa de los valores de la investigación aplicada es mirar hacia el porvenir, buscar iniciativas innovadoras, priorizar aquellos proyectos que tengan mayor 
capacidad de futuro, aprender de la experiencia internacional, pero sobre todo buscar la mayor innovación posible.

12. Foco en Nuevas Formas de Turismo: el foco de la investigación turística debe estar en las nuevas formas de turismo. La nuevas modalidades de turismo según el Código Universal de Ética son: El turismo de naturaleza y el ecoturismo se reconocen como formas de turismo particularmente enriquecedoras y valorizadoras, siempre que respeten el patrimonio natural y la población local y se ajusten a la capacidad de ocupación de los lugares turísticos (OMT, 2001:8). A partir de la ética humanista que compartimos, buscar que el ser humano desarrolle formas de turismo sustentadas en motivaciones saludables, de crecimiento personal, de desarrollo social, intelectual, deportivo o recreativo, es la mejor forma de abrir camino hacia un turismo verdaderamente humanista.

\section{ORIENTACIONES PARA LA INVESTIGACIÓN TURÍSTICA EN LA PATAGONIA}

Prioridad nacional a la Región de Magallanes en Turismo de Intereses Especiales

La Región de Magallanes y Antártica chilena cuenta con extraordinarios parajes naturales, que la convierten en una de las zonas con mayor potencial turístico del país, con trazos ideales para ser focalizada hacia el Turismo de Intereses Especiales. Este privilegio deriva de combinar una vasta superficie de Patagonia, con su flora y fauna endémica, con una variedad de fiordos, mares interiores, porciones insulares y peninsulares, golfos, canales, estuarios $e$ islas, zonas quebradas y montañosas y amplias llanuras y estepas en más de trece millones de hectáreas de superficie, la más extensa del país, con alrededor del $57 \%$ de la superficie territorial regional formando parte del sistema de áreas silvestres protegidas del Estado.

El turismo de esta región, contribuye hoy día con un poco más del siete por ciento del Producto Interno Bruto Regional, involucrando al mismo tiempo alrededor de un $10 \%$ de la fuerza de trabajo ocupada en la región, es decir, ha llegado incluso a sobrepasar sectores tradicionales, otrora más gravitantes en la economía. Este antecedente pone de relieve la incidencia que está llamado a ejercer con su potencial en la economía regional, y hace honor al lema que lo distingue como fuerza impulsora de la lucha contra la pobreza, de creación de empleo y de armonía social.

Una zona con tan alto potencial turístico es al mismo tiempo una región del planeta que posee condiciones excepcionales para la investigación científica del turismo y al mismo tiempo requiere de esta investigación para alcanzar un verdadero desarrollo sustentable del sector.

Orientación y temas del futuro de la investigación turística en Patagonia

La investigación turística en Magallanes y la macro zona sur austral debe ser "investigación aplicada". Esta clase de enfoque concibe a la investigación como:

- vinculada a las necesidades declaradas de los actores del quehacer turístico, tanto privados como públicos;

- inserta en las políticas, planes y programas de desarrollo formulados para los distintos ámbitos del sector;

- ligada al ámbito territorial propio de las Universidades y Centros de Investigación regionales;

- participativa, integrando a los diversos agentes de la actividad turística;

- que se traduzca en políticas y programas de desarrollo que impulsen la actividad;

- formativa, esto es, que fortalezca las competencias del capital humano regional.

Las temáticas de la investigación turística del futuro están estrechamente vinculadas con los acontecimientos de la economía mundial y los cambios en las exigencias de calidad de vida de las sociedades modernas y de los viajeros. Se pueden visualizar algunas áreas de investigación de gran interés y con oportunidades para los investigadores.

- ampliación y mejoramiento de las estadísticas turísticas básicas de interés regional y local;

- estudios sobre la calidad de los servicios y de la oferta de los Centros Turísticos de Patagonia;

- estudios acerca de los mercados interna- 
cionales para el turismo de Patagonia.

- incorporación de nuevas áreas al movimiento turístico y las perspectivas que se abren para desarrollar estudios en Antártica y el territorio subantártico son altamente significativos.

- estudios de impacto ambiental de proyectos turísticos y de efectos sobre el turismo de proyectos de otros sectores económicos, tales como gas, petróleo, etc.

- prospectiva y evaluación del efecto de las crisis y los desastres naturales.

- prospección y evaluación de recursos de los ecosistemas de la Patagonia para el desarrollo turístico;

- estudios sobre los Recursos Humanos necesarios para la actividad turística;

- estudios tendientes al ordenamiento territorial y a la definición de áreas prioritarias para el desarrollo turístico y su consiguiente planificación;

- aspectos de la salud humana tanto síquica como física que tengan relación con el Turismo y la recreación;

- búsqueda de nuevas opciones turísticas (Ecoturismo, Turismo Científico, de Navegación, Turismo Rural, de Aventura, entre otros) son algunos de los temas del futuro más importante para la investigación en Patagonia.

Existen importantes desafíos y oportunidades para desarrollar Investigación e Innovación y Transferencia Tecnológica en Turismo en las regiones australes, dado que es un sector económico emergente con grandes perspectivas de desarrollo en la zona y la Universidad de Magallanes (en adelante UMAG.) tiene la oportunidad y la responsabilidad de liderar la fundamentación científica del sector y la formación de capital humano para el desarrollo sustentable del turismo en la Patagonia.

La iniciativa de establecer un Programa de Investigación Científica en Turismo en Patagonia se ha visto respaldada por la propuesta de SOCIETUR Chile en orden a solicitar que: El Estado, a través de la Corporación de Fomento de la Producción, impulse la creación de Consorcios destinados a desarrollar Investigación e Innovación en turismo, los que deberán coordinar su actuación en inves- tigaciones estadísticas con el Instituto Nacional de Estadísticas y el Servicio Nacional de Turismo. Se preferirá la creación de Consorcios en regiones turísticas y en Universidades y Centros de investigación acreditados (SOCIETUR, 2008:7). Además, la Sociedad de Investigadores en Turismo de Chile solicitó que: El Gobierno de Chile, a través de la Comisión Nacional de Investigación Científica y Tecnológica impulse la creación de un área de investigación específica en materia de Turismo de Intereses Especiales en los fondos de investigación e innovación actualmente existentes (SOCIETUR, 2008:7).

Programa de Investigación y Desarrollo en

Turismo de la Universidad de Magallanes

La visión del Centro de Investigación en Turismo de la UMAG es articular un Programa Internacional de Investigación, Desarrollo e Innovación (I+D+i) en Turismo de Intereses Especiales de la Patagonia, que se constituya en un referente científico en esta área de conocimiento a nivel nacional y latinoamericano, capaz de generar teorías del turismo, con sólidas bases científicas, metodologías homogéneas, series temporales y sostenidas en el tiempo y que realice estudios comparados a nivel regional, nacional e internacional. Sus atributos diferenciadores principales están basados en la cooperación con universidades y centros de $\mathrm{I}+\mathrm{D}$ nacionales e internacionales y en su especialización en el turismo de intereses especiales y en sus extraordinarios recursos naturales, culturales e históricos de relieve mundial y el estado de desarrollo de su industria turística con clara vocación científica.

Este programa de investigación, desarrollo e innovación en turismo de la Patagonia, que lidera la UMAG, se encuentra enfocado en el análisis del comportamiento del turismo internacional hacia Patagonia y especialmente del TIE (ecoturismo, turismo de naturaleza, turismo cultural, científico, entre otros). Se caracteriza además por el fomento del trabajo interdisciplinario, involucrando diferentes ciencias como la ecología, historia, antropología, arquitectura, economía, administración, ingenierías, entre otras. Su ámbito territorial de actuación se centra en tres grandes ecosistemas de relieve mundial para la conservación y para el futuro del planeta: Patagonia, Antártica y la región Subantártica. Apuesta 
por el desarrollo de subsectores turísticos propios de la macroregión y alternativos a los tradicionales, como la hotelería no convencional, las empresas tour operadoras locales y agencias de viajes, los cruceros y el turismo náutico, la administración y gestión de áreas silvestres protegidas, los museos y centros de investigación, los centros de montaña y centros deportivos en general, entre otros. El enfoque de investigación aplicada que debe fortalecer el programa se realiza coordinadamente con el sector público (gobiernos locales y regionales), el sector privado (emprendimientos) y organizaciones no gubernamentales relevantes al desarrollo del turismo en los tres ecosistemas de su hábitat natural. Este programa debe enfocarse especialmente en potenciar el desarrollo de las empresas familiares y en las pymes y en la generación de alianzas estratégicas para el cumplimiento de sus objetivos de consolidar la investigación, el desarrollo y la innovación en una de las regiones turísticas más importantes del mundo. Al mismo tiempo, la investigación en turismo en Patagonia debe tener un importante vínculo de Extensionismo con el sector productivo, de tal forma que, a través de publicaciones, conferencias, difusión por medios digitales, coloquios, jornadas y seminarios, los profesionales y técnicos, empresarios y directivos de instituciones públicas y organizaciones empresariales puedan utilizar el conocimiento generado para lograr el pleno aprovechamiento con carácter sustentable del inmenso potencial de desarrollo del turismo en Patagonia.

\section{REFLEXIONES FINALES}

El mejoramiento de la calidad de la investigación turística en Chile y en la Región de Magallanes, resulta ser un desafío que se encuentra hoy justo con una encrucijada de un proceso integral de cambios en el sector turismo.

Las Universidades y los Centros de Investigación, en general, se encuentran inmersos en un proceso de reestructuración y modernización que obliga a que todas sus áreas alcancen un nivel de excelencia, tanto en docencia como en investigación y vinculación. El área de Turismo en Patagonia no puede permanecer ajena a esta dinámica, por el contrario como área emergente debe hacer un gran esfuerzo por la calidad.

El desarrollo del sector Turismo nacional ha alcanzado un importante nivel de crecimiento que exige la generación de conocimiento científico y de información técnica capaz de aportar las soluciones y reflexiones necesarias para que ese crecimiento se desenvuelva en condiciones adecuadas para afrontar la creciente competitividad de los destinos turísticos mundiales.

La inserción de las economías nacionales en mercados globales no sólo es una integración del comercio, sino también debe ser una integración educacional y científica. Los investigadores turísticos, en especial los de la Región Austral y Patagonia, no pueden quedar al margen de los procesos de globalización, sino por el contrario, deben ser sus pioneros y sus conciencias críticas.

El desarrollo de diferentes disciplinas científicas que confluyen en el estudio del turismo es muy acelerado y el fenómeno turístico debe ser abordado con las metodologías y técnicas modernas de investigación científica.

El esfuerzo para lograr que la investigación científica del turismo en Magallanes y en países de Latinoamérica supere el desfase respecto a la modernización universitaria, turística, económica, es responsabilidad tanto de los cuadros académicos y en especial del Estado y el sector privado. La sociedad en su conjunto, las autoridades, los empresarios y trabajadores deben comprender el valor de la investigación y aportar los recursos necesarios para que ésta se desarrolle. Será responsabilidad de los académicos, aprovechar óptimamente, con innovación y con verdadera dedicación científica, esos espacios que existen y se vayan generando para la investigación en torno al turismo, en particular en la zona austral llamada a tener un rol patagónico en el desarrollo de esta industria a nivel mundial.

La realización de dos seminarios de difusión de resultados de investigación en turismo, con la participación de destacados investigadores de las mas diversas disciplinas, tanto de UMAG como de CEQUA y Fundación OMORA (2010 y 2011) en Punta Arenas, por parte de la Red de I+D+i en Turismo, constituye uno de los primeros pasos en la dirección de crear un gran Centro Internacional de Ciencia aplicada al Turismo en la zona austral de Chile.

No cabe duda, que sin una inversión fuerte en recursos humanos calificados y dotados de los medios necesarios, no habrá despegue de la investigación 
turística en Patagonia. De ahí que esto represente también un desafío para las instituciones y para los académicos. Un académico en una universidad regional que no está desarrollando investigación y publicando no está cumpliendo con una parte fundamental de su rol y de lo que la comunidad legítimamente espera de él.

El perfeccionamiento de los investigadores jóvenes es un requisito fundamental para consolidar la investigación en Turismo y generar un avance significativo hacia el mejoramiento de la calidad de la investigación. Esto es especialmente cierto, ya que la producción científica se eleva a partir del logro de la categoría de Magíster y Doctorados en la especialidad. La creación del programa de Magister al alero del Centro de $\mathrm{I}+\mathrm{d}+\mathrm{i}$ en Turismo de la UMAG no sólo se reflejará en la cantidad sino especialmente en la calidad de la investigación, ya que precisamente son los estudios de postgrado los que apuntan a la formación de investigadores del más alto nivel.

\section{LITERATURA CITADA}

CORFO. (2008). Programa de Innovación en Turismo. En 1er Foro Nacional de Turismo, Valdivia, Chile.

INE-SERNATUR. (2010). Turismo Informe Anual 2009. $112 \mathrm{pp}$.
Organización Mundial del Turismo. (2001). Código Ético Mundial para el Turismo. 8 p.

SOCIETUR-CHILE. (2008). Indicaciones y recomendaciones al proyecto de Ley de Turismo.

Szmulewicz, P. (1998). Universidad e Investigación Turística en Chile. Turisme em Análise, Sao Paulo, 9 (2):107-122.

Szmulewicz, P. (2008). Ley Marco de Turismo para Chile. Una mirada desde la ciencia y la universidad. En 1er Foro Nacional de Turismo, Valdivia, Chile.

Szmulewicz, P., Szmulewicz, E. \& A. De La Barra, (2008). Sintesis Ley $N^{\circ}$ 1092-355/ que establece un sistema institucional para el desarrollo del turismo. Edición Ministerio de Economía, 4 pp.

Szmulewicz, P. (2011). Los Municipios Turísticos en Chile: un desafío pendiente. Encuentro Federal de desarrollo local: turismo como una oportunidad de desarrollo. Consejo Federal de Inversiones, Argentina, 118-125.

Szmulewicz, P. \& K. Veloso (2012). Orientaciones para la construcción de la Ética del Turismo sustentable. en Las Fronteras del Turismo: Perspectivas de la investigación en Turismo, Ed. Nire Negro.

World Economic Forum (2009). The Travel \& Tourism Competitiveness Report 2009, Managing in a Time of Turbulence. Suiza. 525 pp. 
